Corrigendum

\title{
Erratum to "On spurious and real fluctuations of dynamic functional connectivity during rest"
}

\author{
Nora Leonardi, Dimitri Van De Ville* \\ Institute of Bioengineering, Ecole Polytechnique Fédérale de Lausanne (EPFL), Lausanne, Switzerland \\ Department of Radiology and Medical Informatics, University of Geneva, Geneva, Switzerland
}

The authors would like to correct an error in the derivation of the formula for sliding-window covariance of pure sinusoidal signals that are phaselocked (i.e., when fluctuations are spurious). While the derivation of the second term of Eq. (2) correctly uses the product-to-sum trigonometric identity, there is a missing factor of 2 in the similar derivation of the first term of Eq. (2); i.e., after correction it should read:

$$
\begin{aligned}
\frac{\mathrm{TR}}{w} \sum_{i=n-\Delta}^{n+\Delta} x_{i} y_{i} & =\frac{\mathrm{TR}}{w} \sum_{i=n-\Delta}^{n+\Delta}(\cos (\theta)+\cos (4 \pi f i \mathrm{TR}+\theta)) \\
= & \cos (\theta)+\frac{1}{w} \int_{n-\Delta-1 / 2 \mathrm{TR}}^{n+\Delta+1 / 2 \mathrm{TR}} \cos (4 \pi f t+\theta) d t \\
= & \cos (\theta)+\frac{1}{2 w \pi f} \cos (4 \pi f n \mathrm{TR}+\theta) \sin (2 \pi f 2 \Delta+1 \mathrm{TR}),
\end{aligned}
$$

where we have used $w=(2 \Delta+1) \mathrm{TR}$, and, in addition, we improved the approximation of the sum by integration by slightly changing the bounds as to integrate over the full length of the window. The expression of Eq. (5) for sliding-window covariance then reads:

$$
\begin{aligned}
c_{x y}[n]= & \cos (\theta)+\frac{1}{2 w \pi f} \cos (4 \pi f n \mathrm{TR}+\theta) \sin (2 \pi f(2 \Delta+1) \mathrm{TR}) \\
& -\frac{2}{w^{2} \pi^{2} f^{2}} \cos (2 \pi f n \mathrm{TR}) \cos (2 \pi f n \mathrm{TR}+\theta) \sin ^{2}(\pi f(2 \Delta+1) \mathrm{TR}) .
\end{aligned}
$$

These corrections only change the behavior of sliding-window covariance and sliding-window correlation between phase-locked sinusoidal components where the wavelength is larger than the window length. However, the first crossing of the sliding-window covariance (as function of window length) with the true covariance remains unaltered. Therefore, the conclusions and the proposed rule-of-thumb for estimating dynamic functional connectivity do not change. For completeness, we have included the updated Figs. 1 and 2. The other sections of the paper are not affected by this correction. The updated software has been put online at http://miplab.epfl.ch/software/.

The authors like to thank Dr. Andrew Zalesky and Dr. Michael Breakspear for making them aware of the error and the fruitful discussions that followed.

DOI of original article: http://dx.doi.org/10.1016/j.neuroimage.2014.09.007.

* Corresponding author at: EPFL/IBI-STI, Station 17, 1015 Lausanne, Switzerland.

E-mail address: dimitri.vandeville@epfl.ch (D. Van De Ville). 


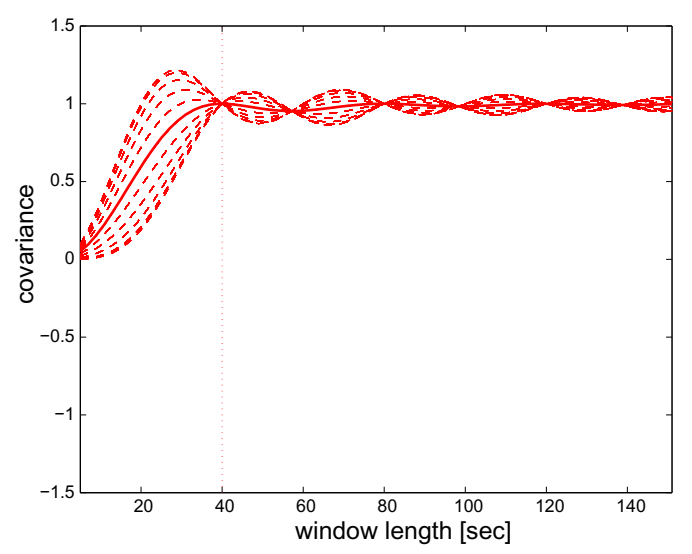

(a)

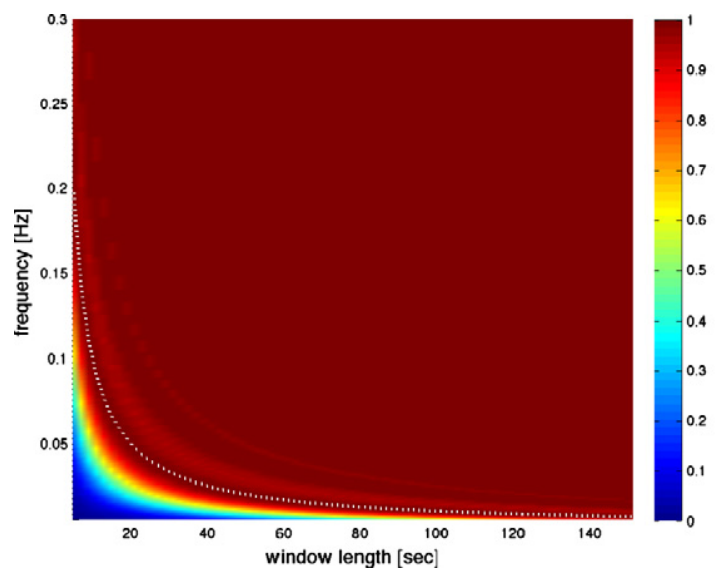

(c)

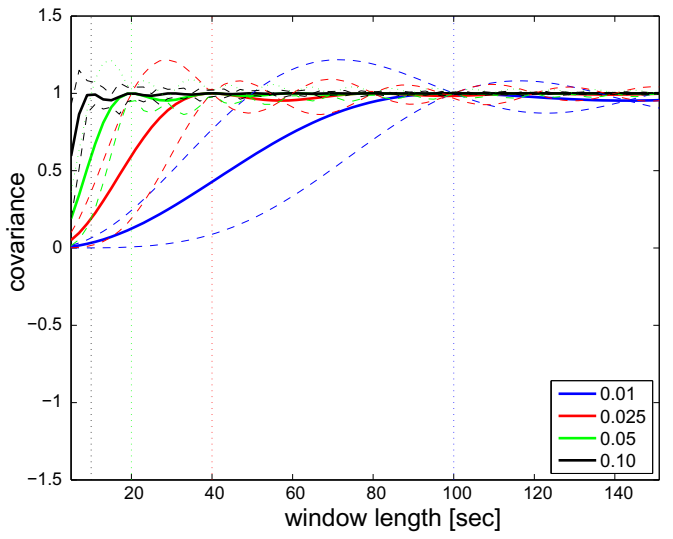

(b)

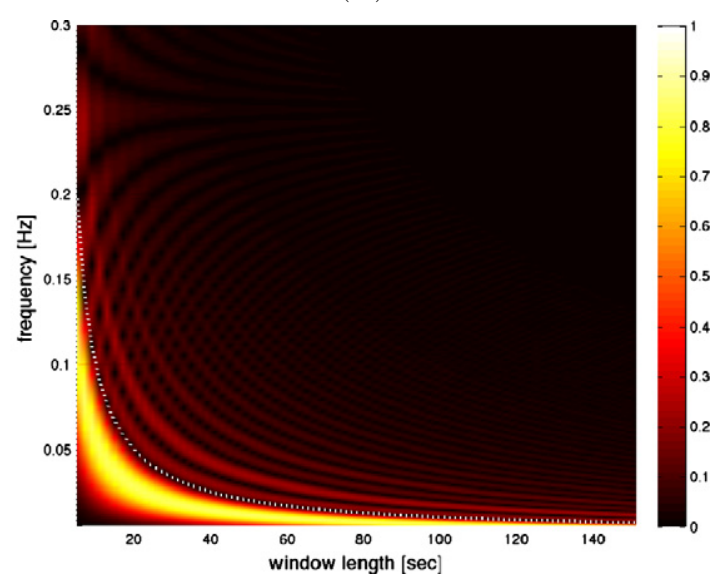

(d)

Fig. 1. Update of Fig. 1

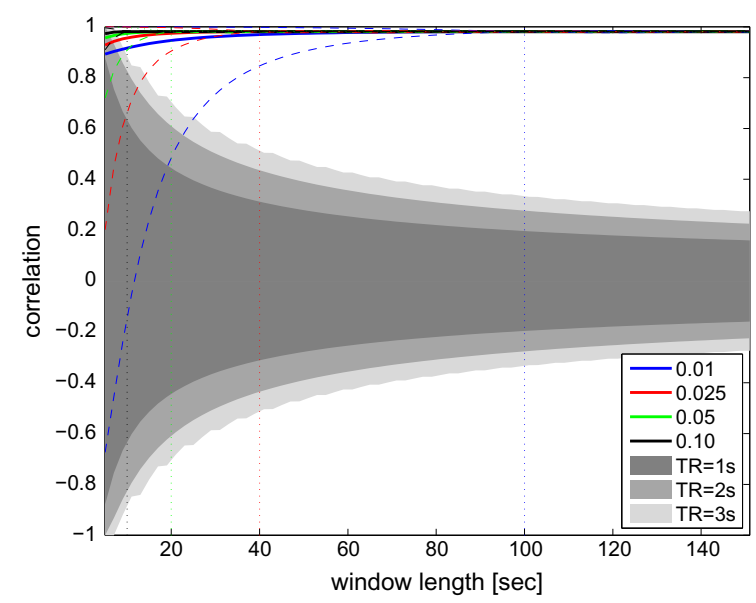

(a)

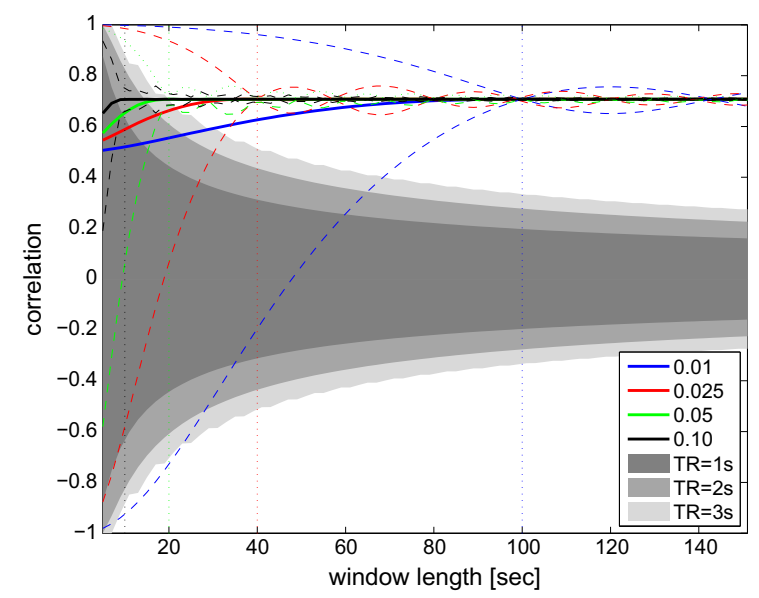

(b)

Fig. 2. Update of Fig. 2. 